\section{Análise da adaptabilidade de carreira em estudantes concluintes do ensino superior}

Amanda Espagolla Santos

Marina Cardoso de Oliveira

\section{RESUMO}

A adaptabilidade de carreira refere-se aos recursos psicossociais que são úteis para lidar com transições de carreira, competência indispensável ao mundo do trabalho contemporâneo. Esta pesquisa objetivou analisar e comparar a adaptabilidade de carreira de universitários concluintes do ensino superior em relação ao sexo, universidade e área de formação. Participaram 387 universitários do último ano da graduação em universidades públicas e privadas de vários cursos e regiões do país. Os instrumentos para a coleta de dados foram a Escala de Adaptabilidade de Carreira e um questionário sociodemográfico. Foram realizadas análises descritivas e comparação entre grupos (ANOVA). Os resultados mostraram bons indicadores em todas as dimensões da adaptabilidade de carreira e diferenças significativas entre grupos, sendo que homens, estudantes da área da saúde e de universidades privadas possuem melhores recursos relacionados ao constructo. Sabendo que a adaptabilidade facilita os processos de transição na carreira, os resultados desta pesquisa podem ser úteis para o planejamento de ações e programas de orientação de carreira para universitários em transição para o trabalho.

Palavras-chave: adaptabilidade de carreira; universitários; transição universidade-trabalho.

\begin{abstract}
Career adaptability refers to the psychosocial resources that are useful to handle with career transitions, essential competence in the contemporary working world. This study aimed to analyze and compare the career adaptability of graduating students of higher education in relation to sex, university and training area. In this research, 387 undergraduates, from public and private universities, from various courses and regions of the country, have participated. The instruments for the data collection were the Career Adaptability Scale and a demographic questionnaire. Descriptive analyses and comparisons between groups (ANOVA) were performed. The results showed good indicators in all dimensions of career adaptability and significant differences between groups, and it was verified that men, health area students and students from private universities have better resources related to the construct. Once it is assumed that adaptability facilitates the career transition processes, the results of this research may be useful for planning actions and programs towards career guidance for college students in transition to work.
\end{abstract}

Keywords: career adaptability, undergraduate students, transition university-work.

\section{Sobre os Autores}

A.E.S

https://orcid.org/0000-00031670-7310

Universidade Federal do Triângulo Mineiro

aespagolla@gmail.com

M.C.O

https://orcid.org/0000-00028715-2853

Universidade Federal do Triângulo Mineiro

mco.uftm@gmail.com

\section{Direitos Autorais}

Este é um artigo de acesso aberto e pode ser reproduzido livremente, distribuído, transmitido ou modificado, por qualquer pessoa desde que usado sem fins comerciais. O trabalho disponibilizado sob a licença Creative Commons CC-BY-NC. 


\section{LZ INTERACÃO EM
PSICOLOGIA}

No Brasil e no mundo, dados apontam para o aumento da quantidade de pessoas que ingressam e concluem o ensino superior (Organisation for Economic Co-operation and Development - OCDE, 2012; Instituto Nacional de Estudos e Pesquisas Anísio Teixeira - INEP, 2017). No Brasil, em 2016, o número de matriculados nas universidades totalizou quase três milhões de ingressantes, sendo que, destes, 82,3\% estavam matriculados em instituições privadas. 0 número de concluintes também ampliou, porém os formandos representam apenas um terço em relação ao número de ingressantes (Instituto Nacional de Estudos e Pesquisas Anísio Teixeira - INEP, 2017.

Assim, a conclusão do ensino superior exige, dos recém-formados, competências para lidar com a transição universidade-trabalho. Ao final da graduação, espera-se que os recém-formados tomem decisões sobre o futuro profissional, que consigam atuar na área de formação, que alcancem seus objetivos individuais e que se adaptem ao trabalho (Wendlandt \& Rochlen, 2008; Oliveira, 2014).

Porém, ao contrário do que é esperado, grande parte dos recém-formados apresenta alguma dificuldade para se inserir no mercado de trabalho. Isso ocorre devido às mudanças tecnológicas e sociais, sendo que a globalização e os avanços das tecnologias impactam na morfologia e nos tipos de contratos de trabalho. Dessa forma, os indivíduos vivenciam grandes desafios nesses momentos de transição, precisando se capacitar para acompanhar as constantes transformações do mercado, da carreira e da sociedade (Silva et al., 2015).

No que diz respeito à carreira, é importante salientar que, nas últimas décadas, o conceito por si só sofreu transformações, deixando de ser uma sequência estável de posições e papeis ocupacionais esperados pelo contexto social para dar lugar aos sentidos que os próprios indivíduos atribuem às suas experiências de vida e de trabalho cada vez mais transitórias, de forma a ser consonante com todos os aspectos de sua vida (Oliveira et al., 2012). Assim, esse fato convida os pesquisadores a adotarem uma visão interdisciplinar e psicossocial para o entendimento da construção da carreira na contemporaneidade (Ribeiro, 2013).

Nessa direção, pesquisas atuais no campo da construção da carreira têm difundido os pressupostos do Modelo da Adaptação de Carreira (Hirschi et al., 2015; Rudolph et al., 2017). Esse modelo busca criar um quadro conceitual para auxiliar as pessoas a desenvolverem recursos psicossociais para lidar com as transições e com as tarefas do desenvolvimento de carreira. O Modelo da Adaptação de Carreira envolve quatro facetas da adaptabilidade, sendo elas: a prontidão adaptativa (adapting); a adaptabilidade de carreira (adaptability); as respostas adaptativas (adapting); e os resultados da adaptação (adaptation). De acordo com - Modelo, a prontidão adaptativa refere-se aos traços psicológicos que indicam a disposição para lidar com as demandas da carreira; a adaptabilidade refere-se aos recursos psicossociais que indicam a capacidade da autorregulação para lidar com a carreira, e, geralmente, é medida em termos de preocupação, controle, curiosidade, e confiança; as respostas adaptativas denotam comportamentos e crenças adaptativas que favorecem a mudança; e, por fim, os resultados da adaptação referemse aos resultados alcançados na carreira, muitas vezes medidos em termos de decisão e compromisso de carreira, bem como satisfação e sucesso no trabalho (Hirschi et al., 2015; Rudolph et al., 2017).

Assim, em um cenário social onde predomina a instabilidade e transitoriedade das trajetórias profissionais, o Modelo da Adaptação de Carreira pode ser útil para auxiliar os indivíduos a se prepararem para lidar com diversas transições ao longo de sua carreira. Entre as diferentes transições pelas quais o indivíduo passará ao longo da construção de sua carreira, uma que se destaca é a transição universidade-trabalho, que pode ser descrita como uma transição antecipada, de entrada para o mercado de trabalho (Oliveira, 2014). Entre os inúmeros atributos individuais que têm potencial para contribuir com sucesso na transição universidade-trabalho, aqueles que promovem a agência pessoal e a adaptabilidade têm se mostrado particularmente significativos (Oliveira, 2014; Hirschi et al., 2015).

Nesse sentido, o constructo da adaptabilidade de carreira tem sido objeto de interesse de muitos pesquisadores, sendo esse conceito um dos mais estudados na atualidade e tornando-se objeto de investigação em diferentes partes do mundo (Savickas et al., 2009/2010; Savickas \& Porfeli, 2012), inclusive no Brasil (Ambiel, 2014; Audibert \& Teixeira, 2015).

Hoje, o conceito organiza-se em torno de quatro dimensões, sendo a preocupação com a carreira, o controle, a curiosidade e a confiança (Audibert \& Teixeira, 2015). A preocupação está relacionada com a orientação ao futuro e ao planejamento no que diz respeito à carreira. 0 controle refere-se às crenças do indivíduo acerca da sua responsabilidade diante do futuro e da sua vontade de agir proativamente em prol de seus objetivos. A curiosidade relaciona-se a comportamentos de caráter exploratório que permitem ao indivíduo ampliar suas perspectivas de ação no cenário ocupacional. E, por fim, 
a confiança refere-se às crenças de capacidade para enfrentar os desafios relacionados com a construção da carreira (Savickas, 2013; Bardagi \& Albanaes, 2015).

No contexto acadêmico, Silveira (2013) concluiu que indivíduos com maior adaptabilidade são aqueles capazes de se preocuparem e possuírem um senso de controle sobre seu futuro vocacional, de lançarem mão da curiosidade para explorarem possíveis identidades e cenários futuros, e de fortalecerem a confiança para alcançarem suas aspirações. Assim, níveis altos de adaptabilidade de carreira seriam capazes de indicar boas condições de lidar com os desafios da transição para o trabalho, aumentar o engajamento acadêmico dos alunos e, potencialmente, contribuir para a permanência e satisfação deles.

Nessa mesma direção, Mognon e Santos (2013) apontaram que o uso de recursos relacionados à adaptabilidade de carreira pode aumentar as oportunidades dos universitários conquistarem emprego após a saída da universidade e alcançarem maior satisfação com o trabalho. Portanto, diante do exposto, observa-se que a adaptabilidade de carreira deve ser considerada como um recurso psicossocial de autorregulação, que depende das condições contextuais e que possibilita desenvolver estratégias e comportamentos de adaptação relacionados ao sucesso, satisfação e estabilidade profissional (Mognon \& Santos, 2013).

Em relação às pesquisas comparativas no contexto acadêmico, há estudos que evidenciam diferenças significativas da adaptabilidade de carreira em relação ao gênero, área de formação e tipo de universidade. Quanto ao gênero, diversos autores apontam que as mulheres possuem baixo otimismo quanto à inserção e possibilidades do mercado, enquanto os homens percebem menos barreiras de carreira e possuem maiores expectativas quanto à remuneração e crescimento profissional (Bardagi \& Hutz, 2012; Bardagi et al., 2003). Por outro lado, há resultados que informam que o sexo não representa um fator de impacto sobre o desenvolvimento da adaptabilidade (Bardagi \& Albanaes, 2015). Nesse sentido, alguns autores argumentam que a não diferença entre sexos na adaptabilidade de carreira é, de certa forma, inconsistente com os resultados obtidos em amostras brasileiras, os quais mostraram maiores índices de controle entre os homens, ou maiores índices de preocupação de carreira entre as mulheres (Silveira, 2013; Bardagi \& Albanaes, 2015). Assim, observa-se que tanto a literatura nacional quanto a internacional não são unânimes em informar sobre as diferenças entre os sexos na adaptabilidade de carreira. Desse modo, vê-se a necessidade de realizar novos estudos que busquem investigar essa relação, trazendo maior compreensão sobre os processos de construção de carreira de homens e mulheres (Bardagi \& Albanaes, 2015).

Já em relação à área de formação, há poucos estudos avaliando tais diferenças, porém há resultados que indicam que estudantes de áreas mais tecnológicas e exatas possuem maiores e melhores expectativas em relação ao mercado do que os de outras áreas (Pitcher \& Purcell, 1998, conforme citado por Bardagi \& Hutz, 2012), mas ainda são necessários outros estudos que confirmem esses dados, principalmente no Brasil. Outro estudo com estudantes concluintes de uma universidade particular, conduzido por Bardagi e Boff (2010), obteve resultados convergentes quanto à área de formação, mostrando que os alunos da área de exatas são mais otimistas que os da área de saúde para realizar a transição universidadetrabalho. Ainda, há estudos que evidenciam que não há diferenças significativas entre essa variável e os níveis de adaptabilidade total, porém há, especificamente, na dimensão preocupação com a carreira, em que estudantes de ciências humanas apresentaram menores escores em comparação com discentes das demais áreas (Bardagi \& Albanaes, 2015).

Não há estudos diretamente relacionados entre adaptabilidade de carreira e tipo de universidade, mas existem autores que apontaram que, da amostra de um estudo, mais da metade dos estudantes das universidades privadas estavam confiantes quanto à meta de conseguir um emprego logo após a formatura (Fleming, 2015). Ainda, encontram-se estudos que verificaram que um nível elevado de adaptabilidade de carreira e vivência acadêmica, como experiências de estágio, proporciona melhores oportunidades para o desenvolvimento de carreira e transição ao papel profissional do indivíduo.

Diante do exposto, ressalta-se a importância dos estudos comparativos no domínio da adaptabilidade de carreira de estudantes universitários, uma vez que tais informações poderão ser úteis para que as universidades realizem processos de intervenção que promovam a adaptabilidade de carreira dos estudantes, podendo influenciar positivamente a construção de carreira durante a transição para o trabalho (Monteiro \& Almeida, 2015; Silva \& Teixeira, 2013). No entanto, os estudos comparativos com essa população são escassos e isso sugere a necessidade de realização de novas investigações com amostras mais significativas e com participantes de diferentes universidades, cursos, sexos e regiões do Brasil (Silva \& Teixeira, 2013; Ambiel et al., 
2016).

Assim, o presente trabalho teve o objetivo de analisar a adaptabilidade de carreira de universitários concluintes, bem como comparar os resultados em relação ao sexo, áreas de formação e tipo de universidade.

\section{MÉTODO}

Trata-se de uma pesquisa quantitativa de natureza descritiva e com recorte transversal. A investigação refere-se a um recorte de um projeto de pesquisa maior intitulado "Transição universidade-trabalho: trajetórias, projetos de vida, identidade profissional e sucesso na carreira".

\section{AMOSTRA}

A amostra foi composta por 387 universitários, de ambos os sexos, vinculados a universidades públicas e particulares, provenientes de vários cursos e regiões do Brasil e que estavam matriculados no último ano da graduação (dois últimos períodos). A idade variou de 18 a 56 anos $(M=24,40$ anos; $D P=5,14)$. Os cursos abrangeram todas as áreas de conhecimento segundo a CAPES, sendo a maioria de ciências da saúde, seguida pelas engenharias, ciências humanas, ciências sociais, ciências exatas e da terra e outros. A maioria da amostra foi proveniente de universidades particulares $(\mathrm{N}=207)$ e do sexo feminino $(\mathrm{N}=235)$.

\section{INSTRUMENTOS}

Para este estudo, construiu-se um caderno de pesquisa contendo os dados pessoais e sociodemográficos dos estudantes, o qual possuía questões como o tipo de universidade em que estuda (pública ou privada), a idade, o sexo, a região do país, a raça e o curso de graduação. Além disso, aplicou-se a versão brasileira da Escala - Escala de Adaptabilidade de Carreira "CareerAdaptAbilities" (Audibert \& Teixeira, 2015; Teixeira et al., 2012;) -, composta por 24 itens que avaliam quatro dimensões da adaptabilidade de carreira: preocupação com a carreira, controle, curiosidade e confiança. A confiabilidade da Escala foi expressa pelo Alfa de Cronbach de 0,94. Os itens podem ser respondidos por meio de uma escala tipo Likert que varia de 1 a 5 , em que 1 corresponde à resposta "Desenvolvi pouco ou nada", e 5 corresponde à resposta “Desenvolvi extremamente bem".

\section{PROCEDIMENTOS DE COLETA E ANÁLISE DE DADOS}

Para realização, este estudo foi submetido e aprovado pelo Comitê de Ética em Pesquisa da Universidade Federal do Triângulo Mineiro, parecer $n^{\circ}$ 1.376.349 (Anexo A).

Após a aprovação do CEP, prosseguiu-se com a coleta de dados, a qual ocorreu de forma presencial (sendo $80,6 \% ; N=312)$ e por meio eletrônico $(19,4 \% ; N=75)$. Os participantes que responderam por meio eletrônico foram contatados por conveniência a partir da rede de contato do grupo de pesquisa, via e-mail e Facebook, e encaminhados para um link onde tiveram acesso ao caderno de pesquisa.

Para a análise, os dados coletados foram tabulados em uma planilha do software IBM SPSS versão 23 , em que foram realizados testes de normalidade, análises descritivas, como o cálculo da frequência e médias, e comparação entre grupos por meio da análise de variância (ANOVA). Optou-se por esse procedimento visto que essa análise produz uma estatística $F$ ou razão $F$ que é semelhante à estatística $t$, pois compara a variância sistemática nos dados com a variância não-sistemática (Field, 2009). Uma vez identificada a diferença entre os grupos, realizou-se o teste post hoc de Tukey e o $d$ de Cohen para conhecer o tamanho do efeito das diferenças, conforme as indicações feitas por Dancey e Reidy (2013).

\section{RESULTADOS E DISCUSSÃO}

0 presente trabalho teve como objetivo analisar a adaptabilidade de carreira de universitários concluintes comparando os resultados em relação a sexo, área de formação e tipo de universidade. Os resultados serão apresentados em duas partes: estudo descritivo das médias dos estudantes nas diferentes dimensões da adaptabilidade de carreira e o estudo comparativo com a análise das médias dos grupos, sexo, área de formação e tipo de universidade.

\section{Estudo Descritivo}

Os universitários concluintes que participaram deste estudo demonstraram possuir um nível de adaptabilidade de carreira médio, tendendo a bom, uma vez que as médias se encontram ligeiramente acima do ponto médio da escala, indicando que possuem os recursos necessários para agregar ao seu desempenho e momento em que se situam. Os resultados mostraram que as médias mais 
altas remeteram às dimensões confiança $(M=4,08$; $D P=$ $0,65)$ e controle $(M=3,99 ; D P=0,68)$.

As dimensões preocupação com a carreira $(M=3,80$; $\mathrm{DP}=0,76)$ e curiosidade $(\mathrm{M}=3,80 ; \mathrm{DP}=0,74)$, embora com escores menores, também apresentaram valores médios tendendo a bom. Tais resultados sugerem que os estudantes concluintes possuem bom nível de recursos psicossociais relacionados a todas as dimensões da adaptabilidade de carreira, em especial nas dimensões de confiança e controle. Discutindo os resultados, tendo em vista a literatura, pode-se dizer que os universitários apresentaram bons indicadores de confiança na carreira (career confidence), possuindo segurança sobre sua capacidade de tomar decisões de carreira com sucesso, o que está ligado à autoestima e autoeficácia, ou seja, crenças de capacidade para enfrentar os desafios relacionados com a construção da carreira (Bardagi \& Albanaes, 2015; Savickas, 2005; Savickas, 2013). 0 controle (career control) está diretamente relacionado ao quanto o sujeito se sente responsável pela construção de sua carreira, demonstrando assertividade nas decisões acerca de sua carreira, autonomia e lócus de controle (Savickas, 2005).

Já a preocupação com a carreira (career concern) foi a terceira maior média e, segundo Savickas (2005), é a dimensão que se relaciona com a preocupação do sujeito com o seu futuro profissional, de modo a orientar e planejálo, o que faz notar a necessidade de estar preparado para lidar com tarefas e transições de carreira. Por fim, a curiosidade (career curiosity) refere-se ao comportamento ativo e exploratório que o sujeito apresenta em busca de conhecimentos e informações sobre si mesmo e sobre a realidade socioprofissional, estando aberto a novas experiências relacionadas à carreira de sua escolha (Bardagi \& Albanaes, 2015; Savickas, 2005; Savickas, 2013). A partir dessas características, pode-se inferir que os universitários dessa amostra apresentaram boas perspectivas de futuro e demonstraram se preocupar com a profissão, procurando possibilidades para uma melhor atuação nesse momento de transição universidadetrabalho. Os resultados também podem refletir um bom planejamento e otimismo em relação ao futuro.

\section{Estudo Comparativo}

Após concluir as análises descritivas, iniciou-se o estudo comparativo para identificar se existiriam diferenças significativas entre as médias dos universitários concluintes em relação ao sexo, tipo de universidade e áreas de formação.

Tabela 1: Comparação entre grupos: Adaptabilidade de carreira em relação ao sexo

\begin{tabular}{|c|c|c|c|c|c|c|c|}
\hline & & & Sexo & & & & \\
\hline Dimensões & $\begin{array}{l}\text { Feminino } \\
\text { Média }\end{array}$ & DP & $\begin{array}{l}\text { Masculino } \\
\text { Média }\end{array}$ & DP & $\mathrm{F}$ & $P$ & $d$ \\
\hline $\begin{array}{l}\text { Adaptabilidade } \\
\text { de Carreira Geral }\end{array}$ & 3,84 & 0,60 & 4,04 & 0,59 & 10,84 & 0,00 & 0,34 \\
\hline $\begin{array}{l}\text { 1. Preocupação } \\
\text { com a carreira }\end{array}$ & 3,73 & 0,76 & 3,92 & 0,75 & 5,56 & 0,01 & 0,25 \\
\hline 2. Controle & 3,90 & 0,69 & 4,13 & 0,65 & 11,43 & 0,00 & 0,34 \\
\hline 3. Curiosidade & 3,73 & 0,73 & 3,91 & 0,76 & 5,53 & 0,01 & 0,24 \\
\hline 4. Confiança & 4,00 & 0,66 & 4,21 & 0,64 & 10,10 & 0,00 & 0,32 \\
\hline
\end{tabular}

Nota. Grau de significância adotado como $p<0,05$

Em relação ao sexo, a Tabela 1 mostra que tanto os homens quanto as mulheres apresentaram um escore médio, tendendo a bom na adaptabilidade de carreira geral e suas dimensões. Esse resultado pode estar atrelado ao atual contexto universitário como estimulador de pensamento crítico, de modo que, possivelmente, haja contato com discussões a respeito da igualdade de gênero, o que leva as mulheres a se envolverem tanto quanto os homens em suas carreiras (Assumpção \& Oliveira, 2018). 
Ainda em relação ao sexo, apesar dos indicadores da adaptabilidade terem sido bons em ambos os sexos, os resultados da comparação entre os grupos (Tabela 1) evidenciaram diferenças significativas quanto à adaptabilidade de carreira geral $[F(1,384)=10,84 ; p=$ $0,00]$, bem como em todas as suas dimensões, sendo: preocupação com a carreira $[F(1,384)=5,56$; $p=0,01]$; controle $[F(1,384)=11,43 ; p=0,00]$; curiosidade $[F(1,384)$ $=5,53 ; p=0,01]$; e confiança $[F(1,384)=10,10 ; p=0,00]$.

Tais resultados indicaram que universitários concluintes do sexo masculino possuíram maiores médias nos indicadores da adaptabilidade de carreira quando comparados com o sexo feminino. Tendo em vista esses resultados, foram realizadas análises para identificar o tamanho do efeito das diferenças. Em relação às médias da adaptabilidade de carreira em geral, o tamanho do efeito foi de intensidade pequena $(d=0,34)$, bem como em todas as suas dimensões de preocupação com a carreira $(d=0,25)$, controle $(d=0,34)$, curiosidade ( $d=$ $0,24)$ e confiança $(d=0,32)$. Essa intensidade mostra que, embora as diferenças nas médias sejam significativas, existe uma grande sobreposição entre os grupos.

Os resultados deste estudo seguem a mesma direção dos resultados encontrados em um estudo com universitários no contexto chinês, que revelaram que os participantes do sexo masculino apresentam resultados ligeiramente superiores em todas as quatro dimensões da adaptabilidade de carreira, contrastando com as estudantes do sexo feminino (Hou et al., 2012). De forma semelhante, o estudo de Silveira (2013) também indicou que os homens apresentaram maiores níveis de adaptabilidade de carreira apenas nas dimensões de confiança ou controle (Fiorini, 2017).

A partir desses resultados, pode-se dizer que, apesar das diferenças encontradas, os resultados convergem para a compreensão de que há diferenças nos escores da adaptabilidade de carreira entre estudantes do sexo masculino e feminino, contudo essas diferenças são de magnitude fraca.

Em relação ao tipo de universidade, também foram observadas diferenças significativas quanto à adaptabilidade de carreira geral $[F(1,384)=15,46 ; p=0,00]$ e suas respectivas dimensões: preocupação com a carreira $[F(1,384)=13,06 ; p=0,00]$; controle $[F(1,384)=5,33 ; p=$ $0,02]$; curiosidade $[F(1,384)=11,42 ; p=0,00]$; e confiança $[F(1,384)=15,83 ; p=0,00]$ (Tabela 2). Evidenciando que, neste estudo, os estudantes concluintes das universidades particulares apresentaram médias maiores nas dimensões de adaptabilidade de carreira do que os universitários das universidades públicas. O tamanho do efeito da diferença entre as médias do constructo de maneira geral dos tipos de universidade foi de intensidade pequena $(d=0,41)$, ou seja, os resultados sugerem que os participantes de universidades privadas são ligeiramente mais preocupados, preparados e com maior estímulo das instituições para vivenciar o momento de transição para a carreira em si. A mesma magnitude de intensidade foi encontrada em preocupação com a carreira $(d=0,36)$, controle $(d=0,24)$, curiosidade $(d=0,34)$ e confiança $(d=0,40)$, sendo que a última dimensão, bem como a adaptabilidade geral, foram as dimensões em que as diferenças entre as médias mais se aproximaram de uma magnitude média.

Não foram identificados estudos que relacionem diretamente a adaptabilidade de carreira com o tipo de universidade em que o sujeito está inserido, porém é sabido que o sistema educacional brasileiro, em todos os níveis, dificilmente disponibiliza suporte aos estudantes no que se refere à educação e construção de carreira, de modo que são poucas as iniciativas desenvolvidas nas instituições de ensino superior que priorizam intervir em questões e problemas relacionados a isso (Jenscheke, 2002). Por outro lado, sabe-se que, de modo geral, as universidades públicas são voltadas para pesquisa, enquanto as particulares direcionam os estudantes mais para a atuação profissional. Nesse sentido, as diferenças encontradas entre os grupos corroboram os achados em outros estudos que apontam que estudantes de universidades privadas possuem maior confiança em relação à transição universidade-trabalho (Fleming, 2015). 
Tabela 2: Comparação entre grupos: Adaptabilidade de Carreira em relação à Universidade

\begin{tabular}{clllllll}
\hline \multicolumn{7}{c}{ Universidade } \\
\hline Dimensões & $\begin{array}{l}\text { Pública } \\
\text { Média }\end{array}$ & DP & $\begin{array}{l}\text { Particular } \\
\text { Média }\end{array}$ & DP & F & P & d \\
$\begin{array}{c}\text { Adaptabilidade } \\
\text { de Carreira Geral }\end{array}$ & 3,79 & 0,57 & 4,03 & 0,61 & 15,46 & 0,00 & 0,41 \\
$\begin{array}{c}\text { 1. Preocupação } \\
\text { com a carreira }\end{array}$ & 3,66 & 0,76 & 3,93 & 0,74 & 13,06 & 0,00 & 0,36 \\
$\begin{array}{l}\text { 2. Controle } \\
\text { 3. Curiosidade }\end{array}$ & 3,90 & 0,67 & 4,06 & 0,69 & 5,33 & 0,02 & 0,24 \\
4. Confiança & 3,94 & 0,72 & 3,92 & 0,75 & 11,42 & 0,00 & 0.34 \\
\hline
\end{tabular}

Nota. Grau de significância adotado como $p<0,05$

Quanto à análise da adaptabilidade de carreira em relação às áreas de formação (Tabela 3), constatou-se diferenças significativas entre os grupos somente nas dimensões de preocupação com a carreira $[F(5,376)=2,88 ; p=0,01]$ e confiança $[F(5,376)=2,71 ; p=0,02]$, sendo as maiores médias dos estudantes da área das ciências da saúde.

Tabela 3: Comparação entre grupos: Adaptabilidade de Carreira em relação à área de formação

\begin{tabular}{|c|c|c|c|c|c|c|c|c|}
\hline Gral & ides Áreas & & & & & & & \\
\hline Dimensões & $\begin{array}{l}\text { Ciências } \\
\text { Exatas e } \\
\text { da Terra } \\
\text { Média }\end{array}$ & $\begin{array}{l}\text { Engenharias } \\
\text { Média }\end{array}$ & $\begin{array}{l}\text { Ciências } \\
\text { da Saúde } \\
\text { Média }\end{array}$ & $\begin{array}{l}\text { Ciências } \\
\text { Sociais } \\
\text { Média }\end{array}$ & $\begin{array}{l}\text { Ciências } \\
\text { Humanas } \\
\text { Média }\end{array}$ & $\begin{array}{l}\text { Outros } \\
\text { Média }\end{array}$ & $F$ & $\mathrm{P}$ \\
\hline $\begin{array}{l}\text { Adaptabilidade } \\
\text { de Carreira Geral }\end{array}$ & 3,78 & 3,90 & 4,02 & 4,00 & 3,80 & 3,73 & 2,13 & 0,06 \\
\hline $\begin{array}{l}\text { 1. Preocupação } \\
\text { com a carreira }\end{array}$ & 3,57 & 3,81 & 3,97 & 3,86 & 3,62 & 3,61 & 2,88 & 0,01 \\
\hline 2. Controle & 3,84 & 3,98 & 4,05 & 4,09 & 3,91 & 3,84 & 0,98 & 0,42 \\
\hline 3. Curiosidade & 3,57 & 3,76 & 3,87 & 3,91 & 3,76 & 3,67 & 0,97 & 0,43 \\
\hline 4. Confiança & 4,13 & 4,06 & 4,21 & 4,15 & 3,93 & 3,80 & 2,71 & 0,02 \\
\hline
\end{tabular}

Nota. Grau de significância adotado como $\mathrm{p}<0,05$

Identificada a existência de diferenças significativas nas médias da adaptabilidade de carreira entre as áreas de formação, utilizou-se o teste post hoc de Tukey para identificar entre quais áreas as médias foram significativamente diferentes. Os resultados indicaram que houve diferença significativa entre as médias dos estudantes da área de ciências da saúde e ciências humanas, em ambas as dimensões. Assim, verificouse que os estudantes da área de ciências da saúde possuíam médias significativamente maiores que os das ciências humanas, tanto na dimensão preocupação com a carreira $(M=3,97 ; D P=0,70, M=3,62 ; D P=0,73$, respectivamente) quanto em confiança ( $M=4,21 ; D P=$ $0,62, M=3,93 ; \mathrm{DP}=0,61$, respectivamente). Não foram 
observadas diferenças significativas entre as demais áreas de formação e dimensões. Ao calcular o efeito da diferença entre as médias (Tabela 4) da dimensão de preocupação com a carreira entre ciências da saúde e ciências humanas, encontrou-se que essa diferença é de intensidade moderada $(d=0,49)$, demonstrando que os estudantes de ciências da saúde tendem a se preocupar mais com o futuro de suas carreiras. 0 efeito da diferença na dimensão de confiança também apresentou a intensidade pequena, tendendo a moderada $(d=0,46)$, indicando uma maior autoeficácia para lidar com a carreira.

Tabela 4: Comparação das médias significativas entre as áreas de formação e cálculo do d de Cohen das dimensões de Preocupação com a Carreira e Confiança.

\begin{tabular}{llllll}
\hline Dimensões & $\begin{array}{l}\text { Ciências da Saúde } \\
\text { Média }\end{array}$ & DP & $\begin{array}{l}\text { Ciências } \\
\text { Humanas }\end{array}$ & DP & $\mathrm{d}$ \\
\hline $\begin{array}{l}\text { Preocupação } \\
\text { com a carreira }\end{array}$ & 3,97 & 0,70 & 3,62 & 0,73 & 0,49 \\
Confiança & 4,21 & 0,62 & 3,93 & 0,61 & 0,46 \\
\hline
\end{tabular}

Os resultados obtidos nessa amostra vão ao encontro do estudo de Bardagi e Albanaes (2015), o qual aponta que não houve diferenças significativas entre os níveis de adaptabilidade total e área de formação, mas, sim, especificamente, na dimensão preocupação com a carreira, em que universitários de ciências humanas mostraram menores escores em comparação com discentes das outras áreas. Pode-se dizer, então, que os estudantes apresentam baixos recursos nessa dimensão, geralmente possuem uma atitude de indiferença diante da carreira, revelando pessimismo e falta de planejamento em relação àqueles com maiores índices de preocupação (Savickas, 2013).

Há, também, estudos relacionados à expectativa dos alunos quanto à transição universidade-trabalho que sugerem que a maior segurança de escolha de carreira é vista em estudantes das áreas de ciências exatas e da saúde, principalmente no curso de medicina - historicamente um curso de grande prestígio e concorrência até os dias atuais, em que os estudantes, em sua maioria, já são mais velhos e melhor preparados para o ingresso no curso -, indicando uma relação de maior estabilidade no mercado de trabalho (Bardagi \& Albanaes, 2015), o que corrobora, de certa forma, os resultados obtidos neste estudo. Apesar disso, há um estudo de Bardagi e Boff (2010) que aponta alunos da área de exatas como sendo mais otimistas que os da área de saúde para realizar a transição universidade-trabalho.

Ainda, Bardagi e Albanaes (2015) ressaltam que há a possibilidade de que as diferenças na adaptabilidade apareçam com maior força à medida que a formação avança, que o contato com a realidade profissional aumenta e as próprias capacidades e limitações são testadas ao longo do curso. Então, é extremamente importante que durante a graduação seja valorizado o desenvolvimento de competências para a liderança, gestão de pessoas e relações interpessoais (Cambiriba et al., 2015).

Para isso, é necessário que as instituições de ensino invistam em uma formação com ênfase em atividades práticas e numa educação que ofereça a construção e aperfeiçoamento de habilidades para que formem profissionais críticos e que sejam capazes de identificar problemas, de forma a alcançar alternativas para superálos com segurança (Barlem et al., 2012; Jesus et al., 2013, citados por Cambiriba et al., 2015). Assim, entende-se que a maneira como cada instituição e/ou cada área promove estratégias de formação e preparação dos alunos para o exercício do trabalho pode influenciar a adaptabilidade futura dos universitários no ingresso ao mundo do trabalho.

\section{CONSIDERAÇÕES FINAIS}

Por meio deste estudo, foi possível realizar uma análise descritiva dos universitários concluintes do ensino superior em relação à adaptabilidade de carreira, 
bem como compará-los em relação ao sexo, tipo de universidade e áreas de formação. Ao analisar os dados, foi possível concluir que os universitários concluintes possuem, de modo geral, bons recursos psicossociais relacionados à adaptabilidade de carreira, apresentando altos índices de confiança e controle, que proporcionam aos estudantes a capacidade para enfrentar os desafios relacionados com a construção da carreira, bem como responsabilidade em relação ao futuro e vontade de ir à busca de seus objetivos (Bardagi \& Albanaes, 2015; Savickas, 2013).

Nesse sentido, há estudos que mostram que estudantes que possuem altos índices associados à adaptabilidade de carreira, de autoestima, proatividade, que se identificam com a área de formação e se engajam na construção da carreira, predizem uma maior empregabilidade e maiores oportunidades para desenvolver a carreira, além de obter sucesso e satisfação nessa transição universidade-trabalho (Ambiel et al., 2016; Hirschi et al., 2015; Ng \& Feldman, 2007; Oliveira, 2014; Rudolph et al., 2017).

Adicionalmente, foi possível concluir que, em relação ao sexo, houve diferenças favorecendo os homens, embora as médias de ambos os sexos estivessem próximas do ponto médio tendendo a bom, tanto na adaptabilidade de carreira total quanto em suas dimensões. Pode-se inferir, então, que, apesar de maiores escores de adaptabilidade de carreira no sexo masculino, a diminuta diferença com o sexo feminino vai de encontro à literatura existente ao considerar que as diferenças entre sexos não são unânimes (Bardagi \& Albanaes, 2015), necessitando de maiores estudos acerca do assunto.

Quanto ao tipo de universidade, foi possível perceber uma diferença significativa entre os universitários concluintes de universidades particulares e públicas. Esses resultados avançam com a compreensão da adaptabilidade da carreira entre estudantes do ensino superior, uma vez que não há, na literatura, relatos de pesquisas que informem sobre essas diferenças. Contudo, as diferenças observadas são semelhantes às encontradas no estudo de Oliveira e Coleta (2008) sobre atitudes de exploração de carreira. Nesta pesquisa, verificou-se que estudantes de universidades particulares tinham escores mais altos nos comportamentos de exploração de carreira do que estudantes de universidades públicas.

Por fim, os resultados relativos às áreas de formação mostraram que estudantes da área da saúde, como medicina, odontologia, fisioterapia etc., possuem maiores escores nas dimensões preocupação com a carreira e confiança do que estudantes das áreas de humanas, de forma a possuírem uma atitude positiva maior frente à carreira, revelando otimismo e planejamento para o futuro.

A partir dos resultados, é possível inferir algumas implicações práticas, inclusive para futuras pesquisas. É importante salientar que esses resultados, de maneira geral, indicam que as instituições formadoras devem ter a responsabilidade de incentivarem o envolvimento dos estudantes em atividades que contribuam para a formação profissional e para o desenvolvimento da carreira após a conclusão da graduação (Oliveira, 2014). Assim, os resultados podem ser úteis para o planejamento de ações de forma contextualizada, como oficinas de orientação acadêmica, maiores oportunidades e possibilidades de atividades extracurriculares, como projetos e estágios, e programas de incentivo para participar além das aulas pré-estabelecidas.

Apesar das contribuições do estudo, algumas limitações podem ser consideradas. Os números discrepantes entre os universitários de cada grande área da CAPES podem ter influenciado o resultado de alguma forma, sendo a grande maioria da área de ciências da saúde, principalmente do curso de medicina, favorecendo sua média em relação às outras áreas. Além disso, o tipo de estudo quantitativo de corte transversal possibilita poucas alternativas de explorar de modo completo a realidade do sujeito. Sugerem-se, ainda, novas pesquisas acerca da influência de universidades públicas e privadas em relação à adaptabilidade de carreira na vida de universitários concluintes, além de aprofundar as diferenças entre áreas de formação e sexo.

Por fim, espera-se que os resultados encontrados contribuam para ampliar a compreensão sobre a importância da adaptabilidade de carreira no processo de transição universidade-trabalho, uma vez que tais recursos implicam em agência pessoal e podem ser úteis para que os concluintes do ensino superior conquistem bons resultados na carreira após a conclusão da graduação.

\section{DECLARAÇÃO DA CONTRIBUIÇÃO DOS AUTORES}

Certificamos que todos os autores participaram 
suficientemente do trabalho para tornar pública sua responsabilidade pelo conteúdo. A contribuição de cada autor pode ser atribuída como se segue:

A. E. S. e M. C. O. foram responsáveis pela investigação da pesquisa; M. C. O. contribuiu com a supervisão, conceitualização, metodologia e revisão da redação do artigo; e A. E. S. realizou a tabulação e análise dos dados, bem como a redação inicial e final do artigo.

\section{DECLARAÇÃO DE CONFLITOS DE INTERESSE}

Os autores declaram que não há conflitos de interesse no manuscrito submetido.

\section{REFERÊNCIAS}

Ambiel, R. A. M. (2014). Adaptabilidade de carreira: uma abordagem histórica de conceitos, modelos e teorias. Revista Brasileira de Orientação Profissional, 15(1), 1524.

Ambiel, R. A. M., Hernández, D. N., \& Martins, G. H. (2016). Relações entre adaptabilidade de carreira e vivências acadêmicas no ensino superior. Psicología desde el Caribe, 33(2), 158-168. http://dx.doi.org/10.14482/ psdc.33.2.7071

Assumpção, M. C. \& Oliveira, M. C. (2018). Estudo do Engajamento com a Carreira em universitários no processo de transição universidade-trabalho. Revista de Psicologia, 9(2), 153-162.

Audibert, A., \& Teixeira, M. A. P. (2015). Escala de adaptabilidade de carreira: evidências de validade em universitários brasileiros. Revista Brasileira de Orientação Profissional, 16(1), 83-93.

Bardagi, M. P., \& Albanaes, P. (2015). Relações entre adaptabilidade de carreira e personalidade: Um estudo com universitários ingressantes brasileiros. Psicologia, 29(1), 35-44. http://dx.doi.org/10.17575/ rpsicol.v29i1.989
Bardagi, M. P., \& Boff, R. M. (2010). Autoconceito, autoeficácia profissional e comportamento exploratório em universitários concluintes. Avaliação: Revista da Avaliação da Educação Superior, 15(1), 41-56. http://dx.doi.org/10.1590/s141440772010000100003

Bardagi, M. P., \& Hutz, C. S. (2012). Mercado de trabalho, desempenho acadêmico e o impacto sobre a satisfação universitária. Revista de Ciências Humanas, 46(1), 183-198. http://dx.doi.org/10.5007/21784582.2012v46n1p183

Bardagi, M. P., Lassance, M. C. P., \& Paradiso, A. C. (2003). Trajetória acadêmica e satisfação com a escolha profissional de universitários em meio de curso. Revista Brasileira de Orientação Profissional, 4(1-2), 153-166.

Cambiriba, T. F. C., Ferronato, A. F., \& Fontes, K. B. (2015). Percepções de egressos de enfermagem frente à inserção no mercado de trabalho. Arquivos de Ciências da Saúde UNIPAR, 18(1), 27-32. http://dx.doi. org/10.25110/arqsaude.v18i1.2014.5155

Dancey, C. P., \& Reidy, J. (2013). Estatísticas em Matemática para Psicologia: Usando SPSS para Windows. Artmed.

Savickas, M. L., Nota, L., Rossier, J., Dauwalder, J., Duarte, M. E., Guichard, J., Soresi, S., Van Esbroeck, R. V., \& van Vianen, A. E. M. V. (2010). A Construção da Vida: Um novo paradigma para entender a carreira no século XXI (Duarte, M. E., Trad.). Revista Portuguesa de Psicologia, 42, 392-406. http://dx.doi.org/10.21631/ rpp42_13 (Trabalho original publicado em 2009)

Field, A. (2009). Descobrindo a estatística usando o SPSS. Artmed.

Fiorini, M. C. (2017). Percepção do funcionamento familiar, diferenciação do self e adaptabilidade de carreira de estudantes universitários. [Dissertação de Mestrado, Universidade Federal de Santa Catarina]. Repositório Institucional UFSC. https://repositorio.ufsc.br/xmlui/ handle/123456789/177764 
Fleming, S. C. R. (2015). Envolvimento acadêmico e autoeficácia na transição para o trabalho: Um estudo com universitários concluintes. [Dissertação de Mestrado, Universidade Federal da Bahia]. Repositório Institucional UFBA. http://repositorio.ufba.br/ri/ handle/ri/21266

Hirschi, A., Herrmann, A., \& Keller, A.C. (2015). Career adaptivity, adaptability, and adapting: A conceptual and empirical investigation. Journal of Vocational Behavior, 87, 1-10. http://dx.doi.org/10.1016/j. jvb.2014.11.008

Hou, Z. J., Leung, S. A., Li, $\quad$ X., Li, $\quad$ X. \& $\mathrm{Xu}, \quad \mathrm{H}$. (2012). Career adapt-abilities scale - China Form: Construction and initial validation. Journal of Vocational Behavior, 80(3), 686-691. http://dx.doi.org/10.1016/j. jvb.2012.01.006

Instituto Nacional de Estudos e Pesquisas Anísio Teixeira - INEP. (2017). Ministério da Educação. Censo da EducaçãoSuperior2016. http://sistemascensosuperior. inep.gov.br/censosuperior_2016/

Jenscheke, B. (2002). Educação Profissional em escolas em uma perspectiva internacional. Em Levenfus, R. S., \& Soares, D. H. P. Orientação Vocacional Ocupacional: Novos achados teóricos, técnicos e instrumentais para a clínica, a escola, a empresa. ArtMed.

Mognon, J. F., \& Santos, A. A. A. (2013). Relação entre vivência acadêmica e os indicadores de desenvolvimento de carreira em universitários. Revista Brasileira de Orientação Profissional, 14(2), 227-237.

Monteiro, S., \& Almeida, L. S. (2015). The relation of career adaptability to work experience, extracurricular activities, and work transition in Portuguese graduate students. Journal of Vocational Behavior, 91, 106-112. http://dx.doi.org/10.1016/j.jvb.2015.09.006

Ng, T. W. H., \& Feldman, D. C (2007). The school-towork transition: a role identity perspective. Journal of Vocational Behavior, 71(1), 114-134. http://dx.doi. org/10.1016/j.jvb.2007.04.004
Oliveira, M. C. (2014). Sucesso na carreira depois da graduação: estudo longitudinal prospectivo da transição universidade-trabalho. [Tese de Doutorado, Universidade de São Paulo]. Biblioteca Digital USP. http://dx.doi.org/10.11606/T.59.2014.tde-13112014101423

Oliveira, M. C., \& Coleta, M. F. D. (2008). Adaptação e validação da Escala Combinada de Atitudes da Maturidade de Carreira (CDA): Versão para estudantes universitários. Revista Brasileira de Orientação Profissional, 9(2), 45-65.

Oliveira, M. C., Melo-Silva, L. L., \& Coleta, M. F. D. (2012). Pressupostos teóricos de super: Datados ou aplicáveis à psicologia vocacional contemporânea. Revista Brasileira de Orientação Profissional, 13(2), 223-234.

Organisation for Economic Co-operation and Development (OECD). (2012). Education at a glance 2012: OECD Indicators. OECD Publishing. https://www.oecdilibrary.org/education/education-at-a-glance-2012_ eag-2012-en

Ribeiro, M. A. (2013). Reflexiones epistemológicas para la orientación professional em América Latina: Una propuesta desde el construccionismo social. Revista Mexicana de Orientación Educativa, 10(24), 2-10.

Rudolph, C. W., Lavigne, K. N., \& Zacher, H. (2017). Career adaptability: A meta-analysis of relationships with measures of adaptivity, adapting responses, and adaptation results. Journal of Vocational Behavior, 98, 17-34. http://dx.doi.org/10.1016/j.jvb.2016.09.002

Savickas, M. L. (2005). The theory and practice of career construction. Em S. Brown, e R. Lent (Eds.), Career development and counselling: Putting theory and research to work (pp. 42-70). Wiley.

Savickas, M. L. (2013). Career construction theory and practice. Em R. W. Lent \& S. D. Brown (Eds.), Career development and counselling: Putting theory and research to work (2nd ed., pp. 147-183). Wiley. 
Savickas, M. L., \& Porfeli, E. J. (2012). Career AdaptAbilities Scale: Construction, reliability, and measurement equivalence across 13 countries. Journal of Vocational Behavior, 80(3), 661-673. http:// dx.doi.org/10.1016/j.jvb.2012.01.011

Silva, A. M. S., Oliveira, M. E. S., \& Oliveira, R. P. A. (2015). Jovens administradores e o mercado de trabalho. Revista ciências humanas e sociais, 2(1), 39-52.

Silva, C. S. C. da, \& Teixeira, M. A. P. (2013). Experiências de estágio: Contribuições para a transição universidadetrabalho. Paidéia (Ribeirão Preto), 23(54), 103-112. http://dx.doi.org/10.1590/1982-43272354201312

Silveira, A. A. (2013). Escala de adaptabilidade de carreira: evidências de validade e fidedignidade em uma amostra de universitários brasileiros. [Dissertação de Mestrado Universidade Federal do Rio Grande do Sul]. https://lume.ufrgs.br/handle/10183/95379
Teixeira, M. A. P., Bardagi, M. P., Lassance, M. C. P., Magalhães, M. O., \& Duarte, M. E. (2012). Career adapt-abilities scale - brazilian form: Psychometric properties and relationships to personality. Journal of Vocational Behavior, 80(3), 680-685. http://dx.doi. org/10.1016/j.jvb.2012.01.007

Wendlandt, N. M., \& Rochlen, A. B. (2008). Adressing the college-to-work transition: Implications for university career counselors. Journal of Career Development, 35(2), 151-165. http://dx.doi. org/ $10.1177 / 0894845308325646$

Data de submissão: 18.11.2018 Data da primeira decisão editorial: 25.07.2019 Aprovado em: 08.08.2019 